\title{
Disseminated Mycobacterium genavense Infection in Two Patients with AIDS
}

\author{
Cecilia D. Gaynor, Robert A. Clark, Franklin P. Koontz, \\ Stefan Emler, Bernard Hirschel, and Larry S. Schlesinger
}

\author{
From the Division of Infections Diseases, Departments of Medicine and \\ Pathology, University of Iowa Hospitals and Clinics. Iowa Citu. Iowa: \\ and the Division of Infectious Diseases, Department of Medicine. Hopilal \\ Cantonal Universitaire, Geneva, Switertand
}

\begin{abstract}
Mycobacterium genavense is a recently defined fastidious organism that has been identified as a cause of disseminated infection in patients with AIDS. We report the cases of two patients who had advanced AIDS and a clinical syndrome of fever, anorexia, abdominal pain, diarrhea, and weight loss. In addition, splenomegaly and lymphadenopathy were prominent in both cases, and in one patient's case radiographic findings were suggestive of splenic abscesses. Mycobacteria isolated from specimens of blood and bone marrow grew in liquid media but not on solid media. The results of DNA probe tests for Mycobacterium tuberculosis and Mycobacterium avium complex were false-positive for both patients. After treatment of the broth cultures to lyse red blood cells, the results of DNA probe tests were negative for these pathogens. Amplification and sequencing of 16S rRNA with use of the polymerase chain reaction indicated that the mycobacterial isolates from both patients had sequences identical to those previously reported for $\boldsymbol{M}$. genavense. One patient survived 5 months after diagnosis, the other 2 months after diagnosis; only one patient responded (transiently) to antimycobacterial chemotherapy.
\end{abstract}

Improvements in antimicrobial therapy have led to increased life-spans among patients infected with the human immunodeficiency virus (HIV). These severely immunocompromised individuals are at increased risk for mycobacterial infections through either reactivation or new acquisition of the microorganisms. Disseminated mycobacterial disease in the late stages of AIDS is most commonly the result of infection with Mycobacterium avium complex (MAC). Despite multidrug therapy, most patients' clinical conditions improve only minimally.

In 1990 Hirschel et al. [1] reported the case of a patient with advanced AIDS who developed a syndrome characterized by fever, anorexia, diarrhea, and weight loss. Staining of biopsies of duodenum and bone marrow and blood buffy coat samples revealed acid-fast bacilli (AFB), which grew in liquid BACTEC medium but not on a variety of solid media. The results of DNA probe tests were negative for Mycobacterium tuberculosis and MAC. Findings of electron microscopy and cell-wall lipid analyses were consistent with a mycobacterium. More recently, Bottger et al. identified additional patients, mostly from Switzerland, with a similar clinical syndrome [2]. Moreover, amplification and sequencing of the $16 \mathrm{~S}$ ribosomal RNA with use of the polymerase chain reaction (PCR) demonstrated a high degree of homology between isolates from these patients and isolates from patients with other mycobacterial species, especially $M$. simiae, as

Received 17 May 1993; revised 27 July 1993.

Reprints or correspondence: Dr. Larry S. Schlesinger, Division of Infectious Diseases. Department of Medicine, University of lowa Hospitals and Clinics, SW 54-14 GH. Iowa City. Iowa 52242.

Clinical Infectious Diseases 1994;18:455-7

(C) 1994 by The University of Chicago. All rights reserved

1058-4838/94/1803-0032\$02.00 well as unique features; these features led to the designation of a new species. Mycobacterium genavense [2].

To date only two small series of cases in which $M$. genavense infection occurred outside of Europe have been published: one series was from the United States [3], the other was from Australia [4]. We report the cases of two patients with $M$. genavense infection who were seen at The University of lowa Hospitals and Clinics, review the published literature on this infection, and highlight the unusual features of this disease. We also reiterate an important potential problem with the use of DNA probes for detection of $M$. tuberculosis and MAC in specimens containing red blood cells.

\section{Case Reports}

Case 1. A 35-year-old homosexual man was found to be seropositive for HIV in 1988. His CD4 lymphocyte count was $150 / \mu \mathrm{L}$, and he received therapy with zidovudine. Apart from a mild bout of Pnounocystis carinit pneumonia and recurrent perirectal ulcers (caused by herpes simplex), his condition was stable until March 1992, when he developed constant abdominal pain. diarrhea, and weight loss. At that point his CD4 lymphocyte count was $82 / \mu \mathrm{L}$. Examination of stool specimens revealed only Giardia cysts, but he failed to respond to two courses of therapy with metronidazole. An abdominal ultrasonogram showed splenomegaly. The patient became anemic, and biopsy of bone marrow revealed $\mathrm{AFB}$ and multiple granulomata. The patient was treated with rifampin, ethambutol, clofazimine. ciprofloxacin, and isoniazid, but his symptoms persisted. Endoscopy revealed candidal esophagitis, and biopsy of the duodenum revealed AFB. A computerized tomography (CT) scan showed hepatosplenomegaly and mesenteric adenopathy. In October 1992 he had changes in his mental status and developed ataxia: a 
CT scan revealed multiple intracerebral lesions that were suggestive of toxoplasmosis. He failed to respond to therapy with pyrimethamine and clindamycin and died 2 weeks later.

Case 2. A 20-year-old heterosexual man was found to be seropositive for HIV in April 1992. One month later he developed abdominal pain, diarrhea, and headache; a 30-lb weight loss was also noted at this time. His CD4 lymphocyte count was $5 / \mu \mathrm{L}$. An abdominal ultrasonogram revealed mild splenic enlargement. Clostridium difficile toxin was detected in specimens of his stool; he received a 2-week course of metronidazole therapy because of diarrhea, which subsequently abated. In June 1992 he developed severe left-upper-quadrant pain, fever, and night sweats, although his CD4 lymphocyte count had risen to $85 / \mu \mathrm{L}$ while he was receiving zidovudine therapy. A CT scan revealed splenic hypodensities and paraaortic lymphadenopathy. A biopsy of bone marrow showed multiple granulomata and occasional AFB.

The patient started receiving therapy with ethambutol, rifampin, ciprofloxacin, and clofazimine. As he experienced adverse effects while receiving therapy with rifampin, therapy with clarithromycin was substituted. His symptoms decreased over the next 2 months, and he began to gain weight. A repeated CT scan revealed homogeneity of both the liver and spleen. In August 1992 he developed a hemorrhagic left pleural effusion. AFB were not seen in the pleural fluid, but white pleural plaques were observed at thoracoscopy and a biopsy revealed noncaseating granulomata. The patient developed respiratory failure postoperatively and died.

\section{Microbiology}

Samples of blood and bone marrow from each patient yielded mycobacteria in BACTEC 13A (blood) or 12B (bone marrow) bottles (Becton Dickinson Diagnostic Instrument Systems, Sparks, MD) in 4-6 weeks. DNA probe analyses (Gen-Probe, San Diego, CA) of both samples from both patients were initially positive for $M$. tuberculosis as well as for MAC. We subsequently ascertained that blood and bone marrow were interfering with the luminescent probe tests (a finding recently reported by Evans et al. [5]), which resulted in false-positive readings. To eliminate this problem, as recommended by those authors, we treated a $1.8-\mathrm{mL}$ aliquot from each positive BACTEC bottle with $0.2 \mathrm{~mL}$ of a $1 \%$ $\mathrm{SDS} / 5 \mathrm{~m} M$ EDTA solution, $\mathrm{pH} 8.0$, and vigorously vortexed it before centrifugation at $15,000 \mathrm{~g}$ for $10 \mathrm{~min}$. The procedure was repeated one time, and ultimately the pellet was washed with $2 \mathrm{~mL}$ of water before the probe assay was performed. DNA probe analysis of samples treated in this manner was negative. We have performed this procedure on samples that are known to be positive, and it does not interfere with true-positive results of DNA probe analysis. In our laboratory all $\mathrm{BACTEC}$ specimens that yield mycobacteria are routinely treated in the above-described manner if they contain blood or bone marrow before the luminescent probe procedure is performed.

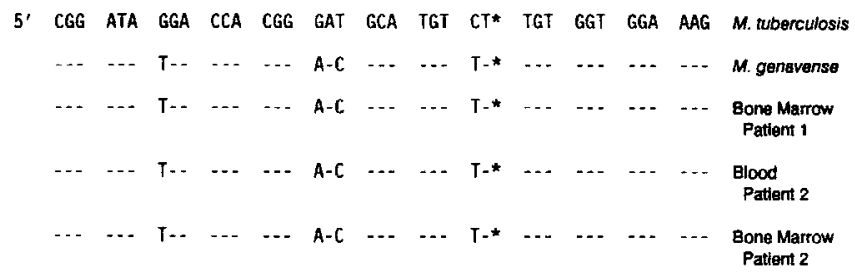

Figure 1. 16S rRNA sequences of $M$. tuberculosis and $M$. genavense from clinical samples. The first nucleotide corresponds to position 176 of Escherichia coli 16S rRNA. Asterisks represent deletions relative to $E$. coli. $M$. mberculosis rRNA is shown as a reference, and only nucleotides of $M$. genavense that are different from those of $M$. tuberculosis are shown.

Although the mycobacteria initially cultured in BACTEC bottles were successfully subcultured in other bottles, no growth was obtained on any solid media despite the use of triplicate sets (incubated at $30^{\circ} \mathrm{C}, 35^{\circ} \mathrm{C}$, and $42^{\circ} \mathrm{C}$ ) of Middlebrook $7 \mathrm{H} 10$ agar, Löwenstein-Jensen agar, and blood agar as well as enriched chocolate agar for the isolation of Mycobacterium haemophilum [6]. Analysis of $16 \mathrm{~S}$ ribosomal RNA was carried out by PCR amplification and sequencing with use of the primers and conditions previously described [2]. The mycobacterial isolates from both patients had sequences identical to those reported for $M$. genavense [2] (figure 1).

\section{Discussion}

As clinical experience with severely immunocompromised AIDS patients has accumulated, more infections due to fastidious environmental mycobacterial species have become apparent. Examples of these organisms include $M$. avium, $M$. intracellulare, $M$. kansasii, $M$. simiae, $M$. haemophilum, and $M$. malmoense; clearly $M$. genavense should be included in this list $[1-4,6-12]$.

In previously reported cases of $M$. genavense infection in AIDS patients, gastrointestinal disease was emphasized; our two cases illustrate the prominence of splenic involvement in these patients. Patient 1 had significant splenomegaly, and for patient 2 the findings on CT scan were highly suggestive of splenic abscesses, which appeared to respond to antimycobacterial therapy. Khalil et al. [13] included $M$. tuberculosis and MAC in the differential diagnosis of splenic abscesses in HIV-positive patients. In the recent series of cases reported by Wald et al. [3], four of seven patients with $M$. genavense infection had splenic involvement; two patients had splenomegaly, one patient's spleen ruptured during splenectomy, and examination of autopsy-obtained specimens of the third patient's spleen revealed AFB. Four of 18 patients with disseminated $M$. genavense infection whose cases were reported by Bottger et al. [2] had splenomegaly, as did two of three children whose cases were recently reported by Nadal et al. [14]. Thus, we believe that splenic enlargement and splenic abscesses are commonly associated with $M$. genavense infection. 
Clinicians should be alerted to the possibility of $M$. genavense infection in AIDS patients who present with a syndrome of fever, anorexia, weight loss, abdominal pain, diarrhea, splenomegaly, and anemia. Laboratory evidence for diagnosis of this infection consists of detection of AFB in blood, bone marrow, or gut tissue specimens as well as the growth of mycobacteria in liquid media and the failure of growth on subculture on solid agar. Liquid cultures (BACTEC 12B or 13A) should be maintained for at least $6-12$ weeks $[15,16]$. Some laboratories have detected limited growth of $M$. genavense on solid media comprised of either Middlebrook 7HII agar supplemented with mycobactin J [15] or Middlebrook 7H10 agar containing charcoal and yeast extract [16]. This growth may require months of incubation. Recently, Bottger et al. [2] described 16S rRNA sequencing for the definitive identification of $M$. genavense. This approach should be considered when fastidious mycobacteria that fail to grow on solid media are encountered.

The difficulties with cultivation of $M$. genavense have precluded characterization of antimicrobial drug susceptibilities, and treatment of $M$. genavense infection has thus been based on knowledge of $M$. avium susceptibilitics. Variable results have been observed with multiple chemotherapeutic regimens $[2,3,14]$. Survival after initiation of therapy for $M$. genavense infection has ranged from 2 months to $>14$ months [ 1,3$]$. One of our patients had little or no response to therapy and died after 5 months (death was caused by another opportunistic infection). The second patient's symptoms decreased while combination therapy was administered, but this patient died after 2 months. A granulomatous pleural process was noted shortly before death. It is difficult to assess whether infection with $M$. genavense contributes substantially to shortened life-spans since most patients who are infected with $M$. genavense have multiple opportunistic infections.

Little is known about the pathogenesis and epidemiology of $M$. genavense infection. Hirschel et al. [1] documented bacteria within the cytoplasm of histiocytes in pathology specimens and demonstrated growth of bacteria in vitro within mouse peritoneal macrophages. Because of the prominence of gastrointestinal disease, it has been suggested that infection is acquired via an oral route [1]. Although most cases of $M$. genavense infection in patients with AIDS identified to date have occurred in central Europe, the geographic range of the organism is unknown. Our cases together with those recently reported from Seattle [3] bring the total number of documented $M$. genavense infections in the United States to nine. Nothing is known about the ecology of this mycobacterium, although a recent report indicated that infection due to $M$. genavense occurs in pet birds [17]. Future studies of $M$. genavense infection should be directed at defining the epidemiology and mode of acquisition of this patho- gen as well as at determining rapid methods of diagnosis and techniques for assessing antimicrobial drug susceptibilities.

\section{Acknowledgment}

The authors thank Deb Nollen for her editorial assistance.

\section{References}

1. Hirschel B. Chang HR. Mach N. et al. Fatal infection with a novel, unidentified mycobacterium in a man with the acquired immunodeficiency syndrome. N Engl J Med 1990:323:109-13.

2. Bottger EC. Teske A. Kirschner P, et al. Disseminated "Mrohackinim genavense" infection in patients with AIDS. Lancet 1992:340:76-80.

3. Wald A, Coyle MB. Carlson IC. Thompson RL. Hooton TM. Infection with a fastidious mycobacterium resembling Mrobacterium simiat in seven patients with AIDS. Ann Intern Med 1992:117:586-9.

4. Jackson KM, Russ BC. Sievers A, Dwyer B. A fastidious strain of Micobasterium tuburulosis in a patient with AIDS [letter]. N Engl J Med 1992:326: 1434-5.

5. Evans KD. Nakasone AS. Sutherland PA. de la Maza LM. Peterson EM. Identification of Merobaterium luberculosis and Mrobacerium arim-M. intracellulare directly from primary BACTEC cul tures by using acridinium-ester-labeled DNA probes. J (lin Microbiol 1992:30:2427-31.

6. Dever LL, Martin JW, Seaworth B, Jorgensen JH Varied presentations and responses to treatment of infections caused by Mroharterim haemophilum in patients with AllOS. Clin Infect Dis 1992:14:1195200.

7. Chaisson RE. Moore RD. Richman DI). Keruly J, Greagh T. Incidence and natural history of Mreobactorium arim-complex infections in patients with advanced human immunodeficiency virus disease treated with zidovudine. Am Rev Respir I)is 1992; 146:285-9.

8. Massenkeil G, Opravil M. Salfinger M, von Graevenitz A. Lüthy $R$. Disseminated coinfection with Mrohacteritm avium complex and Mycobacterium hansasii in a patient with AIIDS and liver abscess [letter]. Clin Infect Dis 1992:14:618-9

9. Carpenter JL. Parks JM. Mrobacterium hansasii infections in patients positive for human immunodeficiency virus. Rev Infect IDis 1991:13:789-96.

10. Gordon SM. Blumberg HM. Mrohacterium kansasii brain abscess in it patient with AIDS [letter]. Clin Infect Dis 1992:14:789-90.

11. Becherer P. Hopfer RI. Infection with Micobac/oritum hacmophilum [letter]. Clin Infect Dis 1992:14:793.

12. Zaugg M. Salfinger M. Opravil M. Lüthy R. Extrapulmonary and disseminated infections due to Mrobactorium malmoconse: case report and review. Clin Infect Dis 1993:16:540-9.

13. Khalil T. Uzoaru I. Nadimpalli V. Wurtz R. Splenic tuberculous abscess in patients positive for human immunodeficiency virus: report of two cases and review [letter]. Clin Infect Dis 1992:14:1265-6.

14. Nadal D, Caduff R, Kraft R. et al. Invasive infection with Mrobucte rizm genaverse in three children with the acquired immunodeficiency syndrome. Eur J Clin Microbiol Infect Dis 1993:12:37-43.

15. Coyle MB. Carlson LC. Wallis CK. el al. Laboratory aspects of " M/robacterium genavense," a proposed species isolated from AIISS paticnts. J Clin Microbiol 1992;30:3206-12.

16. Jackson K. Sievers A, Ross BC, Dwyer B. Isolation of a lastidious Mycobacterium species from two AIDS patients. I (lin Microbiol 1992:30:2934-7.

17. Hoop RK. Botger EC. Ossent P. Salfinger M. Mycobacteriosis due to Mvobacterium genavense in six pet hirds. I (Cin Microbiol $1993 ; 31: 990-3$ 